DOI: $10.17516 / 1997-1397-2021-14-3-360-368$

УДК 517.55

\title{
Analytic Continuation of Diagonals of Laurent Series for Rational Functions
}

\author{
Dmitry Yu. Pochekutov* \\ Siberian Federal University \\ Krasnoyarsk, Russian Federation
}

Received 11.12.2020, received in revised form 01.01.2021, accepted 25.03.2021

\begin{abstract}
We describe branch points of complete $\boldsymbol{q}$-diagonals of Laurent series for rational functions in several complex variables in terms of the logarithmic Gauss mapping. The sufficient condition of non-algebraicity of such a diagonal is proven.
\end{abstract}

Keywords: diagonals of Laurent series, hyperplane amoeba, logarithmic Gauss mapping, zero pinch, monodromy.

Citation: D. Yu. Pochekutov, Analytic Continuation of Diagonals of Laurent Series for Rational Functions, J. Sib. Fed. Univ. Math. Phys., 2021, 14(3), 360-368.

DOI: 10.17516/1997-1397-2021-14-3-360-368.

\section{Introduction and preliminaries}

We use the notation $\mathbb{C}^{\times}$for the one-dimensional complex torus $\mathbb{C} \backslash\{0\}$. For vectors $\boldsymbol{z}=$ $=\left(z_{1}, \ldots, z_{n}\right)$ in $\mathbb{C}^{n}$ or $\left(\mathbb{C}^{\times}\right)^{n}$ and $\boldsymbol{\alpha}=\left(\alpha_{1}, \ldots, \alpha_{n}\right)$ in $\mathbb{Z}^{n}$, denote by $\boldsymbol{z}^{\boldsymbol{\alpha}}$ the monomial $z_{1}^{\alpha_{1}} \ldots z_{n}^{\alpha_{n}}$.

Consider a Laurent series for a rational function $F(\boldsymbol{z})=\frac{P(\boldsymbol{z})}{Q(\boldsymbol{z})}$ of $n$ complex variables centered at the origin:

$$
F(z)=\sum_{\alpha \in \mathbb{Z}^{n}} C_{\boldsymbol{\alpha}} z^{\alpha} .
$$

Let $L \subset \mathbb{Z}^{n}$ be a sublattice of the $n$-dimensional integer lattice. Then the generating function for the subsequence $\left\{C_{\boldsymbol{\alpha}}\right\}_{\boldsymbol{\alpha} \in L}$ of the coefficients indexed by $L$ is called the complete diagonal of the Laurent series (1). Throughout the paper, we consider the sublattice of rank 1 generated by the irreducible vector $\boldsymbol{q}=\left(q_{1}, \ldots, q_{n}\right)$ from $\mathbb{Z}^{n} \backslash\{0\}$. We will call the corresponding diagonal

$$
d_{\boldsymbol{q}}(t)=\sum_{k=-\infty}^{\infty} C_{\boldsymbol{q} \cdot k} t^{k}
$$

a complete $\boldsymbol{q}$-diagonal of the Laurent series (1). Such a diagonal can be written naturally as a sum of two subseries $d_{\boldsymbol{q}}^{+}(t)$ and $d_{\boldsymbol{q}}^{-}(t)$ with only non-negative and negative powers of $t$, correspondingly. We call them one-sided $\boldsymbol{q}$-diagonals. Clearly, we have the equality $d_{\boldsymbol{q}}(t)=d_{\boldsymbol{q}}^{+}(t)$ in the case of Taylor series. For the unit vector $\boldsymbol{I}=(1, \ldots, 1)$, we denote $d_{\boldsymbol{I}}(t)$ by $d(t)$, and refer to $\boldsymbol{I}$-diagonal simply as a diagonal.

Further, we consider irreducible polynomials $P(\boldsymbol{z})$ and $Q(\boldsymbol{z})$. It is well-known that domains of absolute convergence of power series are logarithmically convex. In the case of the Laurent

\footnotetext{
*dpotchekutov@sfu-kras.ru https://orcid.org/0000-0002-4545-2129

(C) Siberian Federal University. All rights reserved
} 
series (1), it is convenient to use the notion of an amoeba of the denominator $Q(\boldsymbol{z})$ of the rational function $F(\boldsymbol{z})$ in the description of such domains. Recall [1, Section 6.1] that the amoeba of a polynomial $Q$ is the image of a hypersurface $Z^{\times}(Q)$ under the logarithmic mapping $\Lambda:\left(\mathbb{C}^{\times}\right)^{n} \rightarrow \mathbb{R}^{n}$ defined by

$$
\Lambda(\boldsymbol{z})=\left(\log \left|z_{1}\right|, \ldots, \log \left|z_{n}\right|\right),
$$

where $Z^{\times}(Q)$ is defined in the complex torus $\left(\mathbb{C}^{\times}\right)^{n}$ by zeroes of the polynomial $Q$.

The complement $\mathbb{R}^{n} \backslash \mathcal{A}_{Q}$ consists of a finite number of connected components $E$ that are open and convex. The preimages $\Lambda^{-1}(E)$ of these components are domains of absolute convergence for Laurent expansions (1) (centered at the origin) for the rational function $F(\boldsymbol{z})$ (see Section 2).

Amoebas are closely related to the notion of the logarithmic Gauss mapping

$$
\gamma_{Q}: \operatorname{reg} Z^{\times}(Q) \rightarrow \mathbb{C P}^{n-1}
$$

defined as

$$
\gamma_{Q}(\boldsymbol{z})=\left(z_{1} \frac{\partial Q}{\partial z_{1}}(\boldsymbol{z}): \ldots: z_{n} \frac{\partial Q}{\partial z_{n}}(\boldsymbol{z})\right)
$$

in regular points $\boldsymbol{z}$ of the hypersurface $Z^{\times}(Q)$. In fact, the set of critical points of the logarithmic projection $\Lambda: Z^{\times}(Q) \rightarrow \mathbb{R}^{n}$ contains the boundary $\partial \mathcal{A}_{Q}$ and coincides with $\gamma_{Q}^{-1}\left(\mathbb{R} \mathbb{P}^{n-1}\right)$.

The complete $\boldsymbol{q}$-diagonal $d_{\boldsymbol{q}}(t)$ of the Laurent series (1) that converges in the domain $\Lambda^{-1}(E)$ for a rational function $F$ can be represented as the integral (see Section 2)

$$
d_{\boldsymbol{q}}(t)=\frac{1}{(2 \pi \imath)^{n}} \int_{\Gamma} \frac{P(\boldsymbol{z})}{Q(\boldsymbol{z})} \frac{\boldsymbol{z}^{\boldsymbol{q}}}{\boldsymbol{z}^{\boldsymbol{q}}-t} \frac{d z_{1} \wedge \ldots \wedge d z_{n}}{z_{1} \ldots z_{n}}
$$

over the $n$-dimensional cycle $\Gamma=\Lambda^{-1}\left(\boldsymbol{y}_{2}\right)-\Lambda^{-1}\left(\boldsymbol{y}_{1}\right)$ in $\left(\mathbb{C}^{\times}\right)^{n} \backslash\left\{Z^{\times}\left(Q \cdot\left(\boldsymbol{z}^{\boldsymbol{q}}-t\right)\right)\right\}$. The parameter $t$ in the integral representation is chosen so that the amoeba of the polynomial $z^{q}-t$ (that is the hyperplane $\langle\boldsymbol{q}, \boldsymbol{u}\rangle=\log |t|$ with the normal vector $\boldsymbol{q}$ ) divides the component $E$ into two parts, and points $\boldsymbol{y}_{1}, \boldsymbol{y}_{2}$ are chosen from different parts of this partition. The ramification of the complete $\boldsymbol{q}$-diagonal happens when a value of the parameter $t$ is such that the rank of the $n$-dimensional homology group $\left(\mathbb{C}^{\times}\right)^{n} \backslash\left\{Z^{\times}\left(Q \cdot\left(\boldsymbol{z}^{\boldsymbol{q}}-t\right)\right)\right\}$ drops.

Since $E$ is convex, the restriction of a linear function $\langle\boldsymbol{q}, \boldsymbol{u}\rangle$ to the closure of $E$ in $\mathbb{R}^{n}$ attains extreme values on the boundary $\partial E$. Let $\boldsymbol{u}_{0}=\boldsymbol{u}_{0}(\boldsymbol{q})$ be one of the points of the boundary $\partial E$ such that the function specified above attains an extreme value. Then the branch points of $d_{\boldsymbol{q}}(t)$ should be among points of the form $\boldsymbol{p}^{\boldsymbol{q}}$, where $\boldsymbol{p}=\boldsymbol{p}(\boldsymbol{q})$ is a point of the hypersurface $Z^{\times}(Q)$ such that $\Lambda(\boldsymbol{p})=\boldsymbol{u}_{0}$.

The main result of the present paper is the theorem that characterises branch points of diagonals.

Theorem 1. Let the Laurent series (1) for a rational function of $n$ variables converge in the domain $\Lambda^{-1}(E)$, and let $d_{\boldsymbol{q}}(t)$ be its complete $\boldsymbol{q}$-diagonal. If $\boldsymbol{q}=\gamma_{Q}(\boldsymbol{p})$, where the point $\boldsymbol{p}$ is regular for the logarithmic Gauss mapping and $\Lambda(\boldsymbol{p}) \in \partial E$, then

1. In the case $n=2 k$ the point $t_{0}=\boldsymbol{p}^{\boldsymbol{q}}$ is a branch point of finite order 2 of $d_{\boldsymbol{q}}(t)$.

2. In the case $n=2 k+1$ the point $t_{0}=\boldsymbol{p}^{q}$ is a branch point of infinite order (logarithmic branch point) of $d_{\boldsymbol{q}}(t)$.

In the context of enumerative combinatorics (see. [2, Section 6.1]), there is the following hierarchy of generating functions

$$
\{\text { rational }\} \subset\{\text { algebraic }\} \subset\{D-\text { finite }\} .
$$


It was proven in [3] that complete $\boldsymbol{q}$-diagonals of Laurent series for rational functions of two complex variables are algebraic. In expositions that deals with diagonals (see, for instance, [4, Section 2] or [2, Section 6.3]), treatment of the case of more than two variables is limited by pointing at the example of non-algebraic diagonal of the Taylor series for the rational function of three variables.

Since algebraic functions cannot have branch points of infinite order, Theorem 1 gives the sufficient condition of non-algebraicity of a diagonal in the case when the dimension $n$ is odd.

Corollary 1. Let the Laurent series (1) for a rational function of $2 k+1$ variables converge in the domain $\Lambda^{-1}(E)$, and let $d_{\boldsymbol{q}}(t)$ be its complete $\boldsymbol{q}$-diagonal. If $\boldsymbol{q}=\gamma_{Q}(\boldsymbol{p})$, where the point $\boldsymbol{p}$ is regular for the logarithmic Gauss mapping and $\Lambda(\boldsymbol{p}) \in \partial E$, then $d_{\boldsymbol{q}}(t)$ is a non-algebraic function.

\section{Amoebas and integral representation for diagonal}

From the moment of diagonals appeared on the mathematical scene (see [5, p. 280]), the important role in their study was played by integral representations. George Pólya showed the algebraicity of a diagonal of a bivariate rational Taylor series from a particular class in [6]. His proof was based on a representation of the diagonal by an integral over a contour in the complex plane. Exploiting a similar idea it was shown in $[4,7]$ that the diagonal of an analytic power series $F$ in a bidisk $\left\{\left|z_{1}\right|<A,\left|z_{2}\right|<B\right\}$ can be represented as

$$
d(t)=\frac{1}{2 \pi \imath} \int_{|\zeta|=\varepsilon} F\left(\zeta, \frac{t}{\zeta}\right) \frac{d \zeta}{\zeta},
$$

where $\varepsilon=\left(A+\frac{|t|}{B}\right) / 2$. If, in addition, $F$ converges to a rational function, then evaluating the integral by residues gives that the diagonal is algebraic, see [4, Section 2] and [2, Section 6.3].

Further, in [8] it was proved that the $\boldsymbol{q}$-diagonal of the Taylor series for a rational function $F(\boldsymbol{z})=\frac{P(\boldsymbol{z})}{Q(\boldsymbol{z})}$ of $n$ complex variables holomorphic at the origin has the integral representation

$$
d_{\boldsymbol{q}}(t)=\frac{1}{(2 \pi \imath)^{n}} \int_{\Gamma_{\rho}} \frac{P(\boldsymbol{z})}{Q(\boldsymbol{z})} \frac{\boldsymbol{z}^{\boldsymbol{q}-\boldsymbol{I}}}{\boldsymbol{z}^{\boldsymbol{q}}-t} d \boldsymbol{z},
$$

where the cycle $\Gamma_{\boldsymbol{\rho}}=\left\{\boldsymbol{z} \in \mathbb{C}^{n}:\left|z_{1}\right|=\rho_{1}, \ldots,\left|z_{n}\right|=\rho_{n}\right\}$ is chosen so that the closed polydisk $\left\{\left|z_{1}\right| \leqslant \rho_{1}, \ldots,\left|z_{n}\right| \leqslant \rho_{n}\right\}$ contains no poles of the function $F(\boldsymbol{z})$, and $\boldsymbol{\rho}^{\boldsymbol{q}}>|t|$. It will be convenient for us to use the following notation

$$
\omega=\frac{1}{(2 \pi \imath)^{n}} P(\boldsymbol{z}) \boldsymbol{z}^{\boldsymbol{q}-\boldsymbol{I}} d \boldsymbol{z}
$$

In order to describe the integral representation for a complete $\boldsymbol{q}$-diagonal of the Laurent series (1), we list necessary facts about amoebas of polynomials.

Recall that the Newton polytope $\Delta_{Q}$ of a polynomial $Q$ is the convex hull in $\mathbb{R}^{n}$ of the set of exponents of the monomials occuring with non-zero coefficients in $Q$. According to Propositions 2.4-2.6 in [9], on the set $\{E\}$ of connected components of $\mathbb{R}^{n} \backslash \mathcal{A}_{Q}$ there exists an injective order mapping

$$
\nu:\{E\} \mapsto \Delta_{Q} \cap \mathbb{Z}^{n}
$$


such that the dual cone to $\Delta_{Q}$ at the point $\nu(E)$ coincides with the recession cone of the component $E$. Then it follows from this fact that the number of connected components of the amoeba complement is at most equal to the number of integer points in $\Delta_{Q}$ (see [9, Theorem 2.8]). Note that the proof of the injectivity of $\nu$ also establishes that components $E$ are convex in $\mathbb{R}^{n}$.

Corollary 1.6 in [1] says that all centered at the origin Laurent expansions (1) of a rational function $F(\boldsymbol{z})=\frac{P(\boldsymbol{z})}{Q(\boldsymbol{z})}$ are in a bijective correspondence with the connected components $\{E\}$. The sets $\Lambda^{-1}(E)$ are the convergence domains for the corresponding Laurent expansions. If the rational function $F(\boldsymbol{z})$ is holomorphic at the origin, then its Taylor expansion converges in the domain $\Lambda^{-1}(E)$, where $\nu(E)=(0, \ldots, 0)$, and the point $(0, \ldots, 0)$ is a vertice of the Newton polytope $\Delta_{Q}$.

The following proposition from [3] generalizes the integral representation for diagonals of Taylor series that have been mentioned above.

Proposition 1. Let the Laurent series (1) for a rational function of $n$ variables converge in the domain $\Lambda^{-1}(E)$, where $E$ is a connected component of the complement $\mathbb{R}^{n} \backslash \mathcal{A}_{Q}$, and let $\boldsymbol{y}_{1}$, $\boldsymbol{y}_{2}$ are points in $E$ such that the inequality $\left\langle\boldsymbol{q}, \boldsymbol{y}_{1}\right\rangle\left\langle\left\langle\boldsymbol{q}, \boldsymbol{y}_{2}\right\rangle\right.$ holds for a non-zero $\boldsymbol{q} \in \mathbb{Z}^{n}$. Then the complete $\boldsymbol{q}$-diagonal $d_{\boldsymbol{q}}(t)$ of the Laurent series (1) has the integral representation

$$
d_{\boldsymbol{q}}(t)=\int_{\Gamma} \frac{\omega}{Q(\boldsymbol{z})\left(\boldsymbol{z}^{\boldsymbol{q}}-t\right)},
$$

where $\left\langle\boldsymbol{q}, \boldsymbol{y}_{1}\right\rangle<\log |t|<\left\langle\boldsymbol{q}, \boldsymbol{y}_{2}\right\rangle$, and $\Gamma=\Lambda^{-1}\left(\boldsymbol{y}_{2}\right)-\Lambda^{-1}\left(\boldsymbol{y}_{1}\right)$.

\section{Proof of Theorem 1}

Note that the differential form $\omega$ is regular in $\left(\mathbb{C}^{\times}\right)^{n}$, while the differential form in the integral representation $(3)$ is meromorphic in $\left(\mathbb{C}^{\times}\right)^{n}$ with polar singularities on hypersurfaces

$$
S_{1}=Z^{\times}(Q), \quad S_{2}=Z^{\times}\left(z^{q}-t\right)
$$

Let $\boldsymbol{y}_{1}, \boldsymbol{y}_{2}$ be points in $E$ chosen as specified in Proposition 1 . The fibers $\Lambda^{-1}\left(\boldsymbol{y}_{1}\right), \Lambda^{-1}\left(\boldsymbol{y}_{2}\right)$ of the logarithmic projection over these points are $n$-dimensional real tori $\left(\mathbb{C}^{\times}\right)^{n}$ that define classes in the reduced homology group $H_{n}\left(\left(\mathbb{C}^{\times}\right)^{n} \backslash S_{1} \cup S_{2}\right)$ with compact supports.

We want to show that the family $\left\{S_{1}, S_{2}\right\}$ has a so-called quadratic zero-pinch (see [10, Section IV.1]) at the point $\boldsymbol{p}$ for $t=t_{0}$, where $t_{0}=\boldsymbol{p}^{\boldsymbol{q}}$. For this purpose, we introduce new coordinates $\boldsymbol{w}=\left(w_{1}, \ldots, w_{n}\right)$ in the $n$-dimensional torus $\left(\mathbb{C}^{\times}\right)^{n}$.

We first note that since vector $\boldsymbol{q}$ is irreducible, according to the Invariant Factor Theorem (see [11, Theorem 16.6]), there exists an unimodular transformation $A: \mathbb{Z}^{n} \rightarrow \mathbb{Z}^{n}$ that takes vector

$\boldsymbol{q}$ to vector $\boldsymbol{e}_{1}=(1,0, \ldots, 0)$. This transformation induces the diffeomorphism $\left(\mathbb{C}^{\times}\right)^{n} \rightarrow\left(\mathbb{C}^{\times}\right)^{n}$ defined as

$$
w_{1}=\boldsymbol{z}^{\boldsymbol{a}_{1}}, \ldots, w_{n}=\boldsymbol{z}^{\boldsymbol{a}_{n}}
$$

where $\boldsymbol{a}_{j}$ 's are columns of the matrix for the transformation $A$, and $\boldsymbol{a}_{1}=\boldsymbol{q}$. In new coordinates, the hypersurfaces $S_{1}, S_{2}$ are defined by equations

$$
\tilde{Q}(\boldsymbol{w})=0, w_{1}-t=0
$$

correspondingly. 
Next, assume, without loss of generality, that $\tilde{Q}_{w_{1}}(\tilde{p}) \neq 0$, where the point $\tilde{\boldsymbol{p}}=\left(\boldsymbol{p}^{a_{1}}, \ldots, \boldsymbol{p}^{a_{n}}\right)$. Then, by the Implicit Function Theorem, there exists a sufficiently small neighbourhood $U$ of the point $\tilde{\boldsymbol{p}}$ such that $S_{1}$ is defined in $U$ as a graph of some analytic function,

$$
S_{1} \cap U=\left\{\boldsymbol{w} \in U: w_{1}=f\left(w_{2}, \ldots, w_{n}\right)\right\} .
$$

Therefore, the intersection $S_{1} \cap S_{2}$ is defined in $U$ as the zero set of the system

$$
\left\{\begin{array}{l}
w_{1}-f\left(w_{2}, \ldots, w_{n}\right)=0 \\
w_{1}-t=0
\end{array}\right.
$$

From the definition of the logarithmic Gauss mapping (2), it follows that

$$
\gamma_{\tilde{Q}}(\boldsymbol{w})=\left(1:-w_{2} f_{w_{2}}\left(w_{2}, \ldots, w_{n}\right): \ldots:-w_{n} f_{w_{n}}\left(w_{2}, \ldots, w_{n}\right)\right)
$$

for $\boldsymbol{w} \in U$. In particular, $\gamma_{\tilde{Q}}(\tilde{\boldsymbol{p}})=(1: 0 \ldots: 0)$. Since the $(i, j)$-component of the Jacobian matrix of the logarithmic Gauss mapping $\gamma_{\tilde{Q}}$ at the point $\tilde{\boldsymbol{p}} \in U$ has the form

$$
\left(-\tilde{p}_{i} f_{w_{i} w_{j}}\left(\tilde{p}_{2}, \ldots, \tilde{p}_{n}\right)\right)_{i, j}, \quad i, j=2, \ldots, n,
$$

the Jacobian determinant of $\gamma_{\tilde{Q}}$ at $\tilde{\boldsymbol{p}}$ and the Hessian determinant of the function $f\left(w_{2}, \ldots, w_{n}\right)$ at the point $\left(\tilde{p}_{2}, \ldots, \tilde{p}_{n}\right)$ vanish simultaneously. If $\boldsymbol{p}$ is a regular point of $\gamma_{Q}$ then $\tilde{\boldsymbol{p}}$ is a regular point of $\gamma_{\tilde{Q}}$. So the point $\left(\tilde{p}_{2}, \ldots, \tilde{p}_{n}\right)$ is a Morse critical point for the function $f\left(w_{2}, \ldots, w_{n}\right)$, and by the Morse lemma, there exist local coordinates $\left(\tilde{w}_{2}, \ldots, \tilde{w}_{n}\right)$ in a neighbourhood of this point such that $f=\tilde{w}_{2}^{2}+\ldots+\tilde{w}_{n}^{2}+\boldsymbol{p}^{q}$. So the intersection $S_{1} \cap S_{2}$ is given locally by the equation

$$
\tilde{w}_{2}^{2}+\ldots+\tilde{w}_{n}^{2}+\boldsymbol{p}^{\boldsymbol{q}}-t=0 .
$$

Therefore, the family of the hypersurfaces $S_{1}, S_{2}$ has the quadratic zero-pinch at the point $\boldsymbol{p}$ for $t=\boldsymbol{p}^{q}$.

Thus, for the discriminant value $t_{0}=\boldsymbol{p}^{q}$ of the parameter $t$, we have the standart degeneration of type $P_{i}=P_{2}$ (in terms of the notation of [12, Section I.8]). The monodromy operator

$$
\Phi: H_{n}\left(\left(\mathbb{C}^{\times}\right)^{n} \backslash S_{1} \cup S_{2}\right) \rightarrow H_{n}\left(\left(\mathbb{C}^{\times}\right)^{n} \backslash S_{1} \cup S_{2}\right),
$$

defined by a small loop going around $t_{0}$ was calculated in [10, Part IV]. This operator reduces to the standart Picard-Lefschetz formula for the Morse singularity in $\mathbb{C}^{n-i+1}=\mathbb{C}^{n-1}$.

So, by Theorem 2.4 in [10, Part IV], we have that

$$
\Phi([\Gamma])=[\Gamma]+\iota[\Sigma]
$$

where $\iota$ is a non-zero integer, and the class $[\Sigma]$ is defined as follows. According to the Thom Isotopy theorem, the monodromy acts identically outside a sufficiently small neighbourhood $W$ of the point $\boldsymbol{p}$. Let $\sigma$ be the vanishing sphere of the dimension $n-2$ in the intersection of $S_{1} \cap S_{2}$ and $W$. Then $[\Sigma]=i_{*} \delta^{2}[\sigma]$, the homomorphism $i_{*}$ is induced by the inclusion of $W$ into $\left(\mathbb{C}^{\times}\right)^{n}$, and $\delta^{2}: H_{n-2}\left(S_{1} \cap S_{2} \cap W\right) \rightarrow H_{n}\left(W \backslash\left(S_{1} \cup S_{2}\right)\right)$ is 2-iterated coboundary operator of Leray defined in Theorem 2 of [10, Part II].

Note that the Picard-Lefschetz formula also gives us

$$
\Phi([\Sigma])=(-1)^{n-1}[\Sigma] .
$$


Knowing the transformation of $[\Gamma]$ and $[\Sigma]$ by $\Phi$ allows us to continue the integral

$$
d_{\boldsymbol{q}}(t)=\int_{\Gamma} \frac{\omega}{Q(\boldsymbol{z})\left(\boldsymbol{z}^{\boldsymbol{q}}-t\right)}
$$

analytically along a small loop around the point $t_{0}$. Let

$$
q(t)=\int_{\Sigma} \frac{\omega}{Q(\boldsymbol{z})\left(\boldsymbol{z}^{\boldsymbol{q}}-t\right)} .
$$

Then during one traversal of the mentioned loop the integral for $d_{\boldsymbol{q}}(t)$ goes to

$$
d_{\boldsymbol{q}}(t)+\iota q(t)
$$

If the dimension $n=2 k$, the two traversals of the loop give

$$
d_{\boldsymbol{q}}(t)+\iota q(t)+(-1)^{2 k-1} \iota q(t)=d_{\boldsymbol{q}}(t)
$$

So, the point $t_{0}$ is a branch point of order 2 for the diagonal $d_{\boldsymbol{q}}(t)$. If the dimension $n=2 k+1$, the two traversals of the loop give

$$
d_{\boldsymbol{q}}(t)+\iota q(t)+(-1)^{2 k} \iota q(t)=d_{\boldsymbol{q}}(t)+2 \iota q(t) .
$$

In this case, $t_{0}$ is a branch point of infinite order for the diagonal $d_{\boldsymbol{q}}(t)$. The theorem is proved.

\section{The diagonal of the multivariate geometric series}

The purpose of this section is to illustrate Theorem 1 .

Consider the polynomial $L(z)=1-z_{1}-\ldots-z_{n}$. The multivariate geometric series

$$
\frac{1}{L(z)}=\sum_{\alpha \in \mathbb{N}^{n}} \frac{\left(\alpha_{1}+\ldots+\alpha_{n}\right) !}{\alpha_{1} ! \ldots \alpha_{n} !} z^{\alpha}
$$

converges in the domain $\Lambda^{-1}\left(E_{0}\right)$, where $E_{0}$ is the compoment of the complement $\mathbb{R}^{n} \backslash \mathcal{A}_{L}$ that corresponds to the constant term of $L$.

For convenience, we denote the diagonal of this Taylor series by

$$
\mathfrak{d}_{n}(t)=\sum_{k=0}^{\infty} \frac{n k !}{(k !)^{n}} t^{k} .
$$

The logarithmic Gauss mapping $\gamma_{L}: Z^{\times}(L) \rightarrow \mathbb{C P}^{n-1}$ is a birational isomorphism with the inverse given by

$$
z_{j}=\frac{q_{j}}{q_{1}+\ldots+q_{n}}, \quad j=1, \ldots, n
$$

where $\boldsymbol{q}=\left(q_{1}: \ldots: q_{n}\right) \in \mathbb{C P}^{n-1}$. Also, the point $\boldsymbol{p}=\left(\frac{1}{n}, \ldots, \frac{1}{n}\right)$ is projected by the logarithmic mapping $\Lambda$ to the point of the boundary $\partial E_{0}$, so that, by Theorem 1 , the point $t_{0}=\boldsymbol{p}^{\boldsymbol{I}}=1 / n^{n}$ is a branch point for the diagonal $\mathfrak{d}_{n}(t)$, and the type of this branch point depends on the parity of $n$.

We note that

$$
\mathfrak{d}_{2}(t)=\frac{1}{\sqrt{1-4 t}}
$$


by means of the generalized binomial expansion. Thus, the diagonal $\mathfrak{d}_{2}(t)$ is an algebraic function that has a branch point of the order 2 at $t_{0}=\frac{1}{4}$.

In the case $n=3$, the diagonal (4) is represented by the Gaussian hypergeometric function

$$
\mathfrak{d}_{3}(t)={ }_{2} F_{1}\left(\frac{1}{3}, \frac{2}{3}, 1 ; 27 t\right),
$$

so that $t_{0}=\frac{1}{27}$ is a branch point for the diagonal. Note that the parameters of this hypergeometric function are not in Schwarz's list of the cases when the Gaussian hypergeometric function is algebraic.

Proposition 1. The diagonal $\mathfrak{d}_{3}(t)$ has the form

$$
\mathfrak{d}_{3}(t)=a_{3}(t) \log (1-27 t)+b_{3}(t),
$$

in a neighbourhood of the point $t_{0}=\frac{1}{27}$, where the functions $a_{3}(t)$ and $b_{3}(t)$ are holomorphic and non-vanishing at the point $t_{0}=\frac{1}{27}$.

Proof. According to [13, Section 16], we can write the hypergeometric function ${ }_{2} F_{1}\left(\frac{1}{3}, \frac{2}{3}, 1 ; 27 t\right)$ as the integral

$$
-\frac{1}{2 \pi \imath} \frac{1}{\Gamma\left(\frac{1}{3}\right) \Gamma\left(\frac{2}{3}\right)} \int_{-\frac{1}{2}+\imath \mathbb{R}} \Gamma^{2}(-\zeta) \Gamma\left(\frac{1}{3}+\zeta\right) \Gamma\left(\frac{2}{2}+\zeta\right)(1-27 t)^{\zeta} d \zeta
$$

with the meromorphic integrand that has three groups of poles

$$
\xi_{k}=k, \quad \zeta_{k}=-\frac{1}{3}-k, \quad \eta_{k}=-\frac{2}{3}-k, \quad k \in \mathbb{N} \cup\{0\} .
$$

The poles $\xi_{k}$ lie on the complex plane to the right of the integration contour, while the poles $\zeta_{k}$, $\eta_{k}$ lie to the left of it.

Evaluating the integral as the sum of residues in poles $\xi_{k}$ of the first group gives us the desired representation.

Further, it is clear from the representation

$$
\mathfrak{d}_{4}(t)={ }_{3} F_{2}\left(\frac{1}{4}, \frac{1}{2}, \frac{3}{4} ; 1,1 ; 256 t\right)
$$

in the form of the generalized hypergeometric function that the diagonal $\mathfrak{d}_{4}(t)$ has a branch point at $t_{0}=\frac{1}{256}$.

By a happy coincidence, the generalized hypergeometric function ${ }_{3} F_{2}$ that corresponds to this specific set of parameters can be written in the form

$$
\mathfrak{d}_{4}(t)=\left(F\left(\frac{1}{8}, \frac{3}{8} ; 1 ; 256 t\right)\right)^{2}
$$

with a help of Clausen's formula [14]. It allows us to describe a type of the branch point $t_{0}=\frac{1}{256}$ in a way that is similar to the proof of Proposition 1.

Proposition 2. The diagonal $\mathfrak{d}_{4}(t)$ has the form

$$
\mathfrak{d}_{4}(t)=a_{4}(t)(1-256 t)^{\frac{1}{2}}+b_{4}(t),
$$

in a neighbourhood of the point $t_{0}=\frac{1}{256}$, where functions $a_{4}(t)$ and $b_{4}(t)$ are holomorphic and non-vanishing at the point $t_{0}=\frac{1}{256}$. 
Proof. According to [13, Section 16], we can write the hypergeometric function ${ }_{2} F_{1}\left(\frac{1}{8}, \frac{3}{8}, 1 ; 256 t\right)$ as the integral

$$
-\frac{1}{2 \pi \imath} \frac{1}{\Gamma\left(\frac{5}{8}\right) \Gamma\left(\frac{7}{8}\right)} \int_{-\frac{1}{16}+\imath \mathbb{R}} \Gamma(-\zeta) \Gamma\left(\frac{1}{2}-\zeta\right) \Gamma\left(\frac{1}{8}+\zeta\right) \Gamma\left(\frac{3}{8}+\zeta\right)(1-256 t)^{\zeta} d \zeta
$$

where the integration contour separates poles of the function $\Gamma(-\zeta) \Gamma\left(\frac{1}{2}-\zeta\right)$ of the form

$$
\xi_{k}=k, \quad \zeta_{k}=\frac{1}{2}+k
$$

from the poles of $\Gamma\left(\frac{1}{8}+\zeta\right) \Gamma\left(\frac{3}{8}+\zeta\right)$ of the form

$$
\eta_{k}=-\frac{1}{8}-k, \quad \varkappa_{k}=-\frac{3}{8}-k
$$

The parameter $k$ ranges over the set $\mathbb{N} \cup\{0\}$.

We let $b(t)$ denote the sum of residues of the integrand at the point $\xi_{k}$. It occurs that $b(t)$ is holomorphic at $t_{0}=\frac{1}{256}$ and does not vanish at this point. At the same time, the sum of residues of the integrand at the points $\zeta_{k}$ has the form $a(t)(1-256 t)^{1 / 2}$, where the function $a(t)$ is holomorphic at $t_{0}=\frac{1}{256}$ and is non-vanishing at this point.

Thus, the function ${ }_{2} F_{1}\left(\frac{1}{8}, \frac{3}{8}, 1 ; 256 t\right)$ has the representation

$$
a(t)(1-256 t)^{\frac{1}{2}}+b(t)
$$

in some neighbourhood of the point $t_{0}=\frac{1}{256}$. Then the Proposition follows directly from the Clausen formula (5).

The research is supported by grant of the Russian Science Foundation (project no. 20-1120117).

\section{References}

[1] I.Gelfand, M.Kapranov, A.Zelevinsky, Discriminants, resultants and multidimensional determinants, Birkhäuser, 1994.

[2] R.Stanley, Enumerative combinatorics, Volume 2, Cambridge University Press, 1999.

[3] D.Pochekutov, Diagonals of the Laurent Series of Rational Functions, Siberian Math. Journal, 50(2009), no. 6, 1081-1091. DOI: 10.1007/s11202-009-0119-z

[4] H.Furstenberg, Algebraic functions over finite fields, Journal of Algebra, 7(1967), no. 2, 271-277.

[5] H.Poincaré, Les méthodes nouvelles de la mécanique céleste. Tome I, Paris: Gauthier-Villars et fils, 1892 .

[6] G.Pólya, Sur les séries entières, dont la somme est une fonction algébrique, Enseign. Math., 22(1921), 38-47. 
[7] M.L.J.Hautus, D.A.Klarner, The diagonal of a double power series, Duke Math. J., 38(1971), no. $2,229-235$.

[8] D.Ž.Djokovič, A properties of the Taylor expansion of rational function in several variables, J. Math. Anal. Appl., 66(1978), 679-685.

[9] M.Forsberg, M.Passare, A. Tsikh, Laurent Determinants and Arrangements of Hyperplane Amoebas, Advances in Mathematics, 151(2000), no. 1, 45-70.

[10] D.Fotiadi, M.Froissart, J.Lascoux, F. Pham, Applications of an isotopy theorem, Topology, 4(1965), no. 2, 159-191.

[11] C.W.Curtis, I.Reiner, Representation Theory of Finite Groups and Associative Algebras, Interscience Publishers, 1962.

[12] V.A.Vasiliev, Applied Picard-Lefschetz Theory, AMS, 2002.

[13] E.W.Barnes, A new development of the theory of the hypergeometric functions, Proc. Lond. Math. Soc. (2), 6(1908), no. 1, 141-177.

[14] T.Clausen, Ueber die Fälle, wenn die Reihe von der Form $y=1+\ldots$ etc. ein Quadrat von der Form $z=1+\ldots$ etc. hat, Journal für die reine und angewandte Mathematik, 3(1828), 89-91.

\section{Аналитическое продолжение диагоналей рядов Лорана рациональных функций}

Аннотация. Мы описываем точки ветвления полных $\boldsymbol{q}$-диагоналей рядов Лорана рациональных функций нескольких комплексных переменных в терминах логарифмического отображения Гаусса. Доказано достаточное условие неалгебраичности такой диагонали.

Ключевые слова: диагонали рядов Лорана, логарифмическое отображение Гаусса, амеба гиперповерхности, нулевой пинч, монодромия. 